\title{
Precordial T wave Inversions on Electrocardiogram
}

\section{Supreeti Behuria ${ }^{1,4}$, Justin A. Ratcliffe ${ }^{2,4}$, Harry Fischer ${ }^{3,4}$ and Paul Schweitzer ${ }^{2,4}$}

${ }^{1}$ Department of Medicine, Beth Israel Medical Center, USA

${ }^{2}$ Thomas Killip Division of Cardiology, Beth Israel Medical Center, USA

${ }^{3}$ Division of Rheumatology, Beth Israel Medical Center, USA

${ }^{4}$ Beth Israel Medical Center, USA

\begin{abstract}
T wave inversions on an electrocardiogram (EKG) can manifest from any process that result in a relative delay in regional ventricular repolarization. The differential diagnosis of right precordial T wave inversions, leads V1-V3, include myocardial ischemia, a normal athlete's heart, arrhythmogenic right ventricular dysplasia, and pulmonary embolism amongst others. The case discussed below is of a 73 year old man who presented to a cardiologist with chest pain and shortness of breath. He had an initial ECG that showed a normal sinus rhythm with T wave inversions across the right precordial leads. He had an unrevealing coronary angiogram and an echocardiogram that only showed right-sided strain. Thereafter, he had a ventilation perfusion scan, which diagnosed pulmonary embolus. $\mathrm{He}$ was treated with anticoagulation and his symptoms resolved. The present case delineates the importance of recognizing this ECG pattern and, in the correct clinical setting, can facilitate in diagnosis and treatment of a relatively common medical condition.
\end{abstract}

\section{Introduction}

$\mathrm{T}$ wave inversions on an electrocardiogram (EKG) can manifest from any process that result in a relative delay in regional ventricular repolarization. The differential diagnosis of right precordial $\mathrm{T}$ wave inversions, leads V1-V3, include myocardial ischemia, a normal athlete's heart, arrhythmogenic right ventricular dysplasia, and pulmonary embolism amongst others. The case discussed below delineates the importance of recognizing this ECG pattern and, in the correct clinical setting, can facilitate in diagnosis and treatment of a relatively common medical condition.

\section{Case Presentation}

A 73 year-old man with a past medical history of Crohn's disease and hypertension, not currently taking any medications, presents to a cardiologist's office with a complaint of worsening shortness of breath for one month. The patient denied any associated chest pain, cough, fevers, chills, syncope, palpitations, or leg swelling. Prior to the onset of his symptoms, the patient was an avid jogger and had no other physical impairments. Of note, about one month prior, the patient recently drove back to New York from Florida. On physical examination, his temperature was 98.3 degrees Farenheit, blood pressure of 135/78 $\mathrm{mmHg}$, heart rate of 100 beats per minute and oxygen saturation of $98 \%$ on room air. His cardiovascular and lung examination were normal. There was no lower extremity edema and dorsalis pedis pulses were intact bilaterally. Chest $\mathrm{x}$-ray revealed no pathology. Electrocardiogram (Figure 1) showed a normal sinus rhythm with $\mathrm{T}$ wave inversions across the right precordial leads, V1-V3. The patient underwent a left heart catheterization which showed non-obstructive coronary artery disease. A transthoracic echocardiogram revealed normal left ventricular size and function, but a dilated right ventricle with reduced right ventricular function and mild-moderate tricuspid regurgitation with a pulmonary artery systolic pressure of $77 \mathrm{mmHg}$. Subsequently, aventilation perfusion scan showed segmental perfusion/ventilation mismatched defects involving the lateral segments of the lower lobes, medial segment of the right middle lobe, and lingula, which suggested a high probability for pulmonary embolism. A lower extremity doppler also revealed a deep vein thrombosis. The patient was subsequently treated with coumadin, which resulted in an improvement in his clinical status.

\section{Discussion}

$\mathrm{T}$ wave inversions are a common ECG finding in clinical practice and can manifest from any process that results in a relative delay in regional ventricular repolarization [1]. While $\mathrm{T}$ wave inversions in the right precordial leads are a normal finding in children and are considered a juvenile ECG pattern, it is relatively rare to find them in healthy adults. However, when found in adults, $\mathrm{T}$ wave inversions isolated to the right precordial leads, as opposed to T wave inversions in other leads, were not associated with adverse outcomes [2]. Still, there is a broad differential diagnosis of various pathologies that one must consider when evaluating patients with this ECG pattern.

Anteroseptal myocardial ischemia is probably the most common cause of right precordial $\mathrm{T}$ wave inversions in this patient's age group. However, the patient's lack of chest pain, un-evolving ECGs, negative cardiac enzymes, and non obstructive coronary artery disease on cardiac catheterization suggest myocardial ischemia as an unlikely cause of the current ECG pattern.

Next, this ECG finding may simply be a result of the physiologic remodeling of an athlete's heart. A recent review found that $\mathrm{T}$ wave inversions were more prevalent in black male athletes with structurally normal hearts and should be considered a normal variant [3]. To further support this finding, de-training among several athletes resulted in resolution of the $\mathrm{T}$ wave inversions. However, especially in the athlete population, it is important to distinguish normal variants from cardiomyopathies with an increased sudden cardiac death risk. Arrhythmogenic Right Ventricular Dysplasia (ARVD)

${ }^{*}$ Corresponding author: Supreeti Behuria, Beth Israel Medical Center, New York, USA Tel: 773-677-3778; E-mail: sbehuria@chpnet.org

Received November 22, 2013; Accepted December 30, 2013; Published January 07, 2014

Citation: Behuria S, Ratcliffe JA, Fischer H, Schweitzer P (2014) Precordial T wave Inversions on Electrocardiogram. J Gen Pract 2: 142. doi: 10.4172/23299126.1000142

Copyright: $\odot 2014$ Behuria S, et al. This is an open-access article distributed under the terms of the Creative Commons Attribution License, which permits unrestricted use, distribution, and reproduction in any medium, provided the original author and source are credited. 
Citation: Behuria S, Ratcliffe JA, Fischer H, Schweitzer P (2014) Precordial T wave Inversions on Electrocardiogram. J Gen Pract 2: 142. doi: 10.4172/2329-9126.1000142

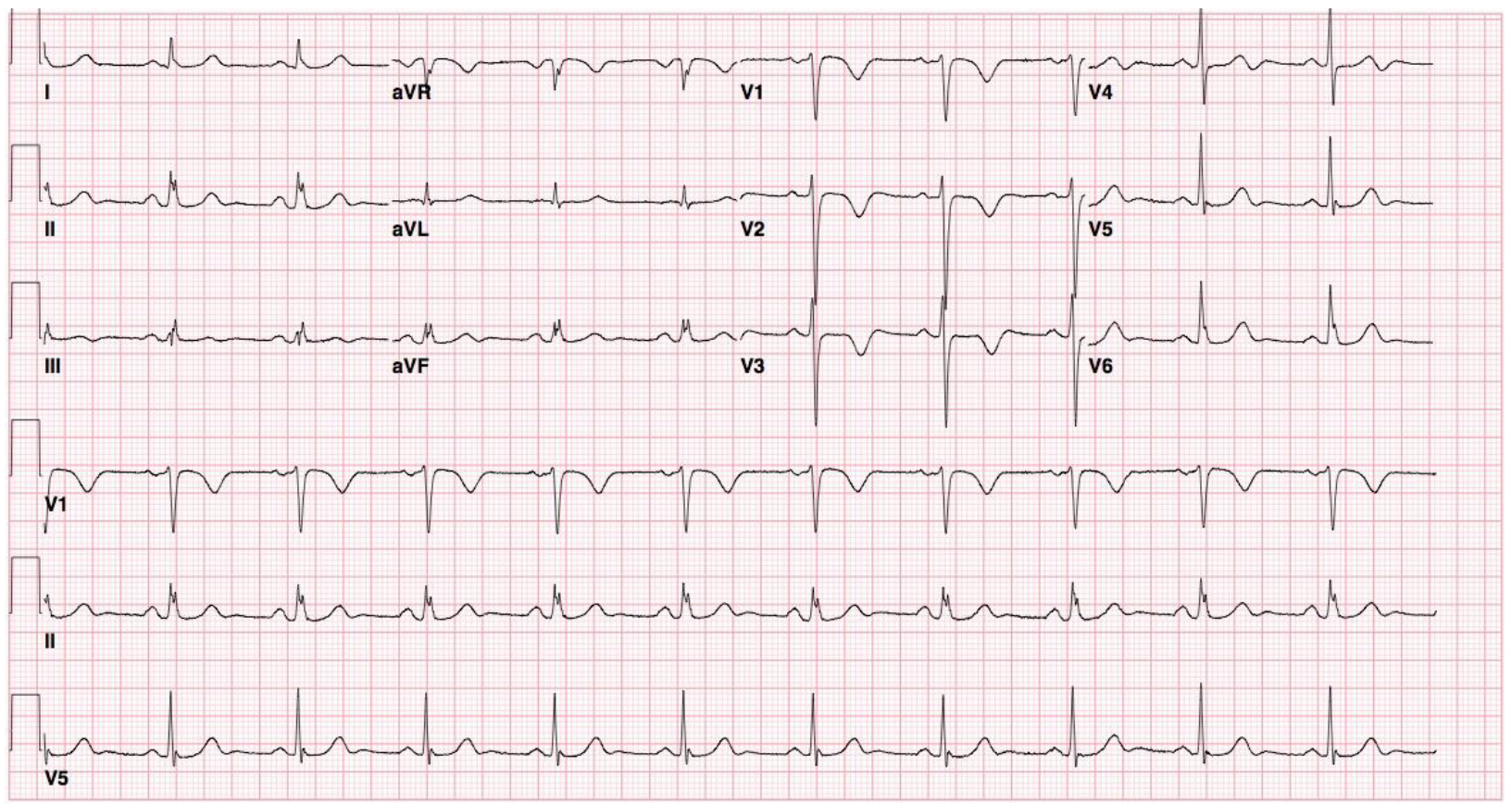

Figure1: Electrocardiogram.

is a cardiomyopathy where fatty and fibrous tissue replaces the right ventricular musculature. The classic ECG findings are postexcitation waves (epsilon waves) in the beginning of the ST segment, but the abnormal repolarization of the right ventricle will lead to $\mathrm{T}$ wave inversions across the right precordial leads as well. The patient's history, clinical presentation, and diagnostic tests do not point toward either a normal athlete's heart or ARVD as the cause of this patient's ECG finding.

Other causes of right precordial $\mathrm{T}$ wave inversions include posttachycardia changes and a post pacemaker $\mathrm{T}$ wave pattern. $\mathrm{T}$ wave inversions can occur following bouts of supraventricular or ventricular tachycardia and will usually be seen in the middle to lateral precordial leads and inferior leads, but may appear in the right precordial leads as well [1]. The post pacemaker $\mathrm{T}$ wave pattern describes $\mathrm{T}$ wave inversions occurring in nonpaced beats following the use of a ventricular pacemaker and appears related to the site of pacemaker stimulation. If the pacemaker lead is placed in the RVOT then $\mathrm{T}$ wave inversion will primarily involve in V1 and V2.

Finally, this patient had a pulmonary embolus (PE), which may also result in an ECG pattern of $\mathrm{T}$ wave inversions in the right precordial leads from right ventricular strain. Pulmonary embolism should always be in the differential when patients present with right precordial $\mathrm{T}$ wave inversions and the corresponding clinical presentation. The present ECG finding is more common than the classic S1Q3T3 pattern [1], also known as the McGinn-White sign, which only occurs in about $30 \%$ of patients with PE [4]. Other ECG findings typical of pulmonary embolism include rightward shift of the QRS axis and transient right bundle branch block [5]. In some unusual cases, ST elevations can occur due to acute right ventricular strain and elevated pressures, usually a result of a submassive or massive PE [4].

\section{Conclusion}

This case emphasizes the utility of recognizing right precordial $\mathrm{T}$ wave inversions on ECG, which can help facilitate in the diagnosis and treatment of a pulmonary embolus.

\section{References}

1. Goldberger A (1991) Myocardial Infarction: Electrocardiographic Differential Diagnosis, 4th ed. St. Louis, Mosby Year Book: 273-320.

2. Aro AL, Anttonen O, Tikkanen JT, Junttila MJ, Kerola T, et al. (2012) Prevalence and prognostic significance of T-wave inversions in right precordial leads of a 12-lead electrocardiogram in the middle-aged subjects. Circulation 125: 25722577.

3. Wilson MG, Sharma S, Carre F, Charron P, Richard P et al. (2012) Significance of deep T-wave inversions in asymptomatic athletes with normal cardiovascular examinations: practical solutions for managing the diagnostic conundrum. $\mathrm{Br} \mathrm{J}$ Sports Med 46: i51-8.

4. Raghav KP, Makkuni P, Figueredo VM (2011) A review of electrocardiography in pulmonary embolism: recognizing pulmonary embolus masquerading as STelevation myocardial infarction. Rev Cardiovasc Med 12: 157-163.

5. Lui CY (1993) Acute pulmonary embolism as the cause of global T wave inversion and QT prolongation. A case report. J Electrocardiol 26: 91-95. 